Dhaka Univ. J. Sci. 60(2): 147-152, 2012 (July)

\title{
A Mathematical Model Related To Chemostat With Variable Yields
}

\author{
S. M. Sohel Rana and Ummi Kulsum \\ Department of Mathematics, University of Dhaka, Dhaka-1000, Bangladesh \\ Email: srana_math@univdhaka.edu
}

Received on 30. 09. 2010. Accepted for Publication on 11. 01. 2012.

\begin{abstract}
In this paper, a three dimensional chemostat model with variable yields is studied. The properties of the steady state points, the local and global stability, the Hopf bifurcation and the positive invariant set for the system are investigated by qualitative analysis of differential equations.
\end{abstract}

Key words: · Continuous culture $\cdot$ Variable yield $\cdot$ Stability $\cdot$ Hopf bifurcation.

\section{Introduction}

Mathematical modeling is a branch of bioscience which is a process that makes a relation between biology and mathematics. The chemostat is both a laboratory device used in biological science, and a well established mathematical model that is important to fields as diverse as ecology, pharmacological compartmental modeling, chemical engineering and medicine. In ecology, it serves as the model of the simple lake where the populations compete for the available nutrient. The analysis of a big number of chemostat models can be found in the monograph Waltman and Smith [10].

Most of the models in chemostat assume that the yield coefficient is a constant. But the accumulation of experimental data suggests that a constant yield fails to explain the observed oscillatory behavior in the chemostat [3]. Cooke [1,2] suggested a linear function for the yield coefficient and declared that a limit cycle may exist in his model. Huang [11,12] and Pilyugin and Waltman [7] constructed the model with a general variable yield, and studied the multiple limit cycles and the hopf bifurcation for the model. However, all these model considered only one microorganism in the system. A three dimensional chemostat with two microorganisms which are both with linear yields was studied by [9]. In the model, the functional reaction functions were in the Monod type, and the yield coefficients were assumed linear function of the concentration of nutrient. The stability of the solution was obtained. Xucheng Huang and Lemin Zhu [5] studied a three dimensional chemostat with quadratic yields. A three dimensional continuous fermentation model with yield coefficients $\delta_{1}=A+B S^{3}$ and $\delta_{2}=C+D S^{4}$ was discussed in [6]. They studied the stability of the equilibrium points, the existence of limit cycles, the hopf bifurcation and the positive invariant set for their model.

In this paper, we study a three dimensional chemostat model of which one yield coefficient is linear and another yield coefficient is cubic functions of the concentration of nutrient. In section II, we formulate the chemostat model with two microorganisms which are with variable yield coefficients and illustrate the process of nondimensionalization to simplify the system. In section III, we study the steady state points with their stability, the positive invariant set, the existence of limit cycles, and the Hopf bifurcation in the two dimensional stable manifold of one microorganism when the other is going to vanish in the competition. In section IV, MATHEMATICA is used for numerical simulation to illustrate the key points of our model. Finally, conclusion of the paper is carried out in section V.

\section{The Model}

At time $t$, let $S(t)$ denote the concentration of nutrient in the vessel, $x(t)$ and $y(t)$ the concentration of two microorganism. The model takes the form

$$
\begin{aligned}
& \frac{d S}{d t}=\left(S_{0}-S\right) Q-\frac{1}{\delta_{1}}\left(\frac{m_{1} S}{k_{2}+S}-L\right) x-\frac{1}{\delta_{2}} \frac{m_{2} S}{k_{2}+S} y \\
& \frac{d x}{d t}=x\left(\frac{m_{1} S}{k_{1}+S}-L-Q\right) \\
& \frac{d y}{d t}=y\left(\frac{m_{2} S}{k_{2}+S}-Q\right) \\
& S(0)>0, x(0)>0, y(0)>0 .
\end{aligned}
$$

where $S_{0}$ is the input concentration of nutrient, $Q$ is the washout rate, $m_{i}$, the maximal growth rates, $k_{i}$, the MichaelisMenton constants, $\delta_{i}$, the yield coefficients and $L$, is the intrinsic consumption rate for the first microorganism, which are all positive. This model is usually called the Monod model or the model with Michaelis Menten dynamics.

Here we investigate the system (1) with yield coefficients $\delta_{1}=A+B S, \delta_{2}=C+D S^{3}$, which means that the production of the microbial biomasses is very sensitive to the concentration of the nutrient.

In system (1) we have used the growth rate functions

$F_{i}(S)=\frac{m_{i} S}{k_{i}+S}$, where $i=1,2$

which have the following common features:

- $F_{i}(0)=0$

- $F_{i}(S)$ is an increasing function of $S$.

- $F_{i}(S)$ approaches a constant value as $S$ approaches infinity.

Performing the standard scaling for the chemostat, let $\bar{S}=\frac{S}{s_{0}}, \bar{x}=\frac{x}{s_{0}}, \bar{y}=\frac{y}{s_{0}}, T=Q t, \overline{m_{l}}=\frac{m_{i}}{Q}, \overline{k_{l}}=\frac{k_{i}}{s_{0}}$,

* Correspondence author : E-mail: srana_math@univdhaka.edu 
$\bar{L}=\frac{L}{Q}$

then drop the bars and replace $T$ with $t$, system (1) becomes

$\frac{d S}{d t}=(1-S)-\frac{1}{A+B S_{0} S}\left(\frac{m_{1} S}{k_{1}+S}-L\right) x-\frac{1}{C+D S_{0}{ }^{3} S^{3}}\left(\frac{m_{2} S}{k_{2}+S}\right) y$

$\frac{d x}{d t}=\left(\frac{m_{1} S}{k_{1}+S}-L-1\right) x$

$\frac{d y}{d t}=\left(\frac{m_{2} S}{k_{2}+S}-1\right) y$

The parameters have been scaled by the operating environment of the chemostat, which are determined by $S_{0}$ and $Q$. The variables are non-dimensional and the discussion is in $R_{+}^{3}=\{(S, x, y) \mid 0 \leq S \leq 1, x \geq 0, \quad y \geq$ $0\}$.

To be biologically meaningful, it is important to prove that the solutions of the model (2), with positive initial data, will remain positive for all time $t>0$. The proof of the following lemma is similar to [8].

Lemma 1: The solutions $S(t), x(t), y(t)$ of (2) are positive for all $t>0$ and for large $t, S(t)<1$.

Lemma 2: For the system (2)

(i) if $m_{1}<L+1$, then $\frac{d x}{d t}<0$ and $\lim _{t \rightarrow \infty} x(t)=0$

(ii) if $m_{2}<1$, then $\frac{d y}{d t}<0$ and $\lim _{t \rightarrow \infty} y(t)=0$.

Proof: Let $F_{1}(S)=\frac{m_{1} S}{k_{1}+S}$. Since $F_{1}{ }^{\prime}(S)>0$ with $F_{1}(0)=$ 0 and $m_{1}=\lim _{S \rightarrow \infty} F_{1}(S)$, we have $L+1-m_{1}>0$. Thus there is $0<\alpha=\min _{t \geq 0}\left\{(L+1)-\max _{0 \leq S<\infty}\left|F_{1}(S)\right|\right\}$ such that $\frac{d x}{d t}<-\alpha x(t)$ for sufficiently large $t$ by the second the equation of (2). This shows $x(t)<x(0) \exp [-\alpha t]$ and since $x(t)>0$ for $t>0$, so we have $\lim _{t \rightarrow \infty} x(t)=0$. This completes the proof (i). Similarly, we can prove (ii).

\section{Steady State Points and Their Stability Analysis}

The system (2) has three steady state points in $R_{+}^{3}$ :

$E_{0}=(1,0,0)$,

$E_{1}=\left(\lambda_{1},\left(1-\lambda_{1}\right)\left(A+B \lambda_{1} S_{0}\right), 0\right)$ and

$E_{2}=\left(\lambda_{2}, 0,\left(1-\lambda_{2}\right)\left(C+D S_{0}^{3} \lambda_{2}^{3}\right)\right)$

where $\lambda_{1}=\frac{k_{1}(L+1)}{m_{1}-(L+1)}$ and $\lambda_{2}=\frac{k_{2}}{m_{2}-1}$ are respectively unique solutions of

$\frac{m_{1} S}{k_{1}+S}-L-1=0$ and $\frac{m_{2} S}{k_{2}+S}-1=0$.

$E_{0}, E_{1}$ and $E_{2}$ will exist if they have non-negative components. The steady state point $E_{0}$ always exists. The other $E_{i}$ 's exist if $0<\lambda_{i}<1(i=1,2)$.

The parameter values $\lambda_{1}, \lambda_{2}$ represent the break-even concentration of the nutrient for the microorganism $x(t)$ and the microorganism $y(t)$ respectively and play an important role in determining competitive ability. With the help of Lemma 1, we can prove the following lemma.

Lemma 3: (i) If $m_{1}>L+1$ and $\lambda_{1}>1$ then $\frac{d x}{d t}<0$ and $\lim _{t \rightarrow \infty} x(t)=0$; (ii) If $m_{2}>1$ and $\lambda_{2}>1$ then $\frac{d y}{d t}<0$ and $\lim _{t \rightarrow \infty} y(t)=0$.

So in order to avoid the microorganisms vanishing, we need to assume that

$$
0<\lambda_{i}<1, \quad i=1,2
$$

(which implies $m_{1}>\left(k_{1}+1\right)(L+1)$ and $m_{2}>k_{2}+1$ )

Theorem 1: Let

$$
D=\left\{\begin{array}{c}
(S, x, y) \mid 0 \leq S \leq l-x-y, \\
0 \leq x \leq\left(A+B S_{0} \lambda_{1}\right)\left(1-\lambda_{1}\right)+\varepsilon_{0}, \\
0 \leq y \leq\left(C+D S_{0}^{3} \lambda_{2}^{3}\right)\left(1-\lambda_{2}\right)+\varepsilon_{0}, \\
0<l<\infty, \varepsilon_{0}>0, \text { constant }
\end{array}\right\} .
$$

Then $D$ is positively invariant under the system (2). In other words, any trajectory initiated $(S, x, y) \in R_{+}^{3}$ enters into $D$ as $t \rightarrow+\infty$.

Proof: By the first equation of (2), any trajectory in $D_{1}=$ $\{(S, x, y) \mid S<0, x>0, y>0\}$ will cross the face $S=0$ into $R_{+}^{3}$. But the trajectory in $D \subset R_{+}^{3}$ will not cross $S=0$ and go to $D_{1}$.

Consider the face $W=S+x+y-l=0 \quad(0<l<+\infty)$ and it is easy to see that

$$
\begin{aligned}
\left.\frac{d W}{d t}\right|_{W=0}= & \left.\left(\frac{d S}{d t}+\frac{d x}{d t}+\frac{d y}{d t}\right)\right|_{S=l-x-y} \\
= & 1-l-x\left(\frac{1}{A+B S_{0}(l-x-y)}-1\right)\left(\frac{m_{1}(l-x-y)}{k_{1}+l-x-y}-L\right)- \\
& y\left(\frac{1}{C+D S_{0}^{3}(l-x-y)^{3}}-1\right)\left(\frac{m_{2}(l-x-y)}{k_{2}+l-x-y}\right) .
\end{aligned}
$$

Since $x, y$ are bounded and $A, B, C, D, S_{0}, m_{i}, k_{i}, i=1,2$ are all positive, $\left.\frac{d W}{d t}\right|_{W=0}<0$ for sufficiently large $l$. Therefore, the trajectory of (2) will cross the face $W=0$ into $D$ from outside to inside. Moreover, both $x=0$ and $y=0$ are the solution faces of the system (2). Thus $D$ is positively invariant under the system (2). The proof of Theorem 1 is completed.

Let

$R_{1}=S_{0} \frac{\left(1-\lambda_{1}\right)\left\{\left(k_{1}+\lambda_{1}\right)^{2}-k_{1} m_{1} \lambda_{1}\right\}-\lambda_{1}\left(k_{1}+\lambda_{1}\right)^{2}}{\left(k_{1}+\lambda_{1}\right)^{2}+\left(1-\lambda_{1}\right) k_{1} m_{1}}$
$R_{2}=S_{0}^{3} \frac{\left(1-\lambda_{2}\right)\left\{3 \lambda_{2}^{2}\left(k_{2}+\lambda_{2}\right)^{2}-k_{2} m_{2} \lambda_{2}^{3}\right\}-\lambda_{2}^{3}\left(k_{2}+\lambda_{2}\right)^{2}}{\left(k_{2}+\lambda_{2}\right)^{2}+\left(1-\lambda_{2}\right) k_{2} m_{2}}$

\section{III.A Local and Global Stability Analysis}

The Jacobian matrix of the system (2) is

$J(S, x, y)=$
$\left[\begin{array}{ccc}T(S, x, y) & -\frac{1}{A+B S_{0} S}\left(\frac{m_{1} S}{k_{1}+S}-L\right) & -\frac{1}{C+D S_{0}{ }^{3} S^{3}}\left(\frac{m_{2} S}{k_{2}+S}\right) \\ \frac{k_{1} m_{1} x}{\left(k_{1}+S\right)^{2}} & \frac{m_{1} S}{k_{1}+S}-L-1 & 0 \\ \frac{k_{2} m_{2} y}{\left(k_{2}+S\right)^{2}} & 0 & \frac{m_{2} S}{k_{2}+S}-1\end{array}\right]$


where,

$T(S, x, y)=-1-\frac{x}{A+B S_{0} S} \frac{k_{1} m_{1}}{\left(k_{1}+S\right)^{2}}+\frac{B S_{0} x}{\left(A+B S_{0} S\right)^{2}}\left(\frac{m_{1} S}{k_{1}+S}-\right.$

$L)-\frac{y}{C+D S_{0}^{3} S^{3}} \frac{k_{2} m_{2}}{\left(k_{2}+S\right)^{2}}+\frac{3 D S_{0}^{3} S^{2} y}{\left(C+D S_{0}^{3} S^{3}\right)^{2}}\left(\frac{m_{2} S}{k_{2}+S}\right)$.

At $E_{0}$, the eigenvalues of $J\left(E_{0}\right)$ are

$$
-1, \frac{m_{1}}{k_{1}+1}-L-1 \text { and } \frac{m_{2}}{k_{2}+1}-1 \text {. }
$$

Hence, $E_{0}$ is stable if $\lambda_{1}>1$ and $\lambda_{2}>1$.

At $E_{1}$, the characteristics equation of $J\left(E_{1}\right)$ is

$\left(r-a_{1}\right)\left(r^{2}+b_{1} r+c_{1}\right)=0$

where

$a_{1}=\frac{m_{2} \lambda_{1}}{k_{2}+\lambda_{1}}-1$

$b_{1}=1+\left(1-\lambda_{1}\right)\left\{\frac{k_{1} m_{1}}{\left(k_{1}+\lambda_{1}\right)^{2}}-\frac{B S_{0}}{A+B S_{0} \lambda_{1}}\right\}$

$c_{1}=\left(1-\lambda_{1}\right) \frac{k_{1} m_{1}}{\left(k_{1}+\lambda_{1}\right)^{2}}>0$.

Therefore, $E_{1}$ is stable iff the roots of (5) have negative real part. By Routh-Hurwitz criteria, the stability holds if and only if $a_{1}<0$ and $b_{1}>0$ or equivalently, $\lambda_{1}<\lambda_{2}$ and $\frac{A}{B}>R_{1}$.

At $E_{2}$, the characteristics equation of $J\left(E_{2}\right)$ is

$\left(r-a_{2}\right)\left(r^{2}+b_{2} r+c_{2}\right)=0$

where

$a_{2}=\frac{m_{1} \lambda_{2}}{k_{1}+\lambda_{2}}-L-1$

$b_{2}=1+\left(1-\lambda_{2}\right)\left\{\frac{k_{2} m_{2}}{\left(k_{2}+\lambda_{2}\right)^{2}}-\frac{3 D S_{0}^{3} \lambda_{2}^{2}}{C+D S_{0}^{3} \lambda_{2}^{3}}\right\}$

$c_{2}=\left(1-\lambda_{2}\right) \frac{k_{2} m_{2}}{\left(k_{2}+\lambda_{2}\right)^{2}}>0$.

Therefore, $E_{2}$ is stable iff the roots of (6) have negative real part. By Routh-Hurwitz criteria, stability holds if and only if $a_{2}<0$ and $b_{2}>0$, or equivalently, $\lambda_{2}<\lambda_{1}$ and $\quad \frac{C}{D}>R_{2}$.

From the above stability analysis of the steady state points of system (2), we can summarize the results in the following theorem.

Theorem 2: The system (2) has three steady state points in $R_{+}^{3}: E_{0}, E_{1}$ and $E_{2}$ in which $E_{0}$ is stable if $\lambda_{1}>1$ and $\lambda_{2}>1$; unstable if $\lambda_{1}<1$ and $\lambda_{2}<1 . E_{1}$ is stable if $A / B>R_{1}$ and $\lambda_{1}<\lambda_{2}$; untable if $A / B>R_{1}$ and $\lambda_{1}>\lambda_{2}$, or $A / B<R_{1} . E_{2}$ is stable if $C / D>R_{2}$ and $\lambda_{1}>\lambda_{2}$; untable if $C / D>R_{2}$ and $\lambda_{1}<\lambda_{2}$ or $C / D<$ $R_{2}$.

Theorem 3: (a) If $\lambda_{1}<\lambda_{2}$, and $A / B>R_{1}$, the steady state point $E_{1}$ is globally asymptotically stable in $R_{+}^{3}$; (b) If $\lambda_{1}>\lambda_{2}$, and $C / D>R_{2}$, the steady state point $E_{2}$ is globally asymptotically stable, too.
Proof: Let

$\Theta=\left\{\begin{array}{c}(S, x, y) \mid 0 \leq S \leq l-x-y, \\ 0 \leq x \leq\left(A+B S_{0} \lambda_{1}\right)\left(1-\lambda_{1}\right)+\varepsilon_{0}, \\ 0 \leq y \leq\left(C+D S_{0}^{3} \lambda_{2}^{3}\right)\left(1-\lambda_{2}\right)+\varepsilon_{0}, \\ 0<l<\infty, \varepsilon_{0}>0\end{array}\right\}$

We first prove that $\Theta$ is positively invariant set of (2).

Consider the face $S=0$, and by equation (2),

$\left.\frac{d S}{d t}\right|_{S=0}=1+\frac{L x}{A}>0$.

Thus, any trajectory in $\{(S, x, y) \mid S<0, x>0, y>0\}$ will go through $S=0$ into $R_{+}^{3}$, but the reverse is not true.

Consider the face $M=S+x+y-l=0(0<l<+\infty)$,

$$
\begin{aligned}
\left.\frac{d M}{d t}\right|_{M=0}= & 1-l-x\left(\frac{1}{A+B S_{0}(l-x-y)}-1\right)\left(\frac{m_{1}(l-x-y)}{k_{1}+l-x-y}-L\right)- \\
& y\left(\frac{1}{C+D S_{0}^{3}(l-x-y)^{3}}-1\right) \frac{m_{2}(l-x-y)}{k_{2}+l-x-y} .
\end{aligned}
$$

Since $x, y$ are bounded and all the parameters are positive, $\left.\frac{d M}{d t}\right|_{M=0}<0$ for sufficiently large $l$. That is, any trajectory in $R_{+}^{3}$ will cross $M=S+x+y-l=0$ into $\Theta$.

Moreover, because both $x=0$ and $y=0$ are the solutions of the system (2). Thus $\Theta$ is positively invariant set of the system (2). In other words, any trajectory initiating in $R_{+}^{3}$ will go to $\Theta$ when $t \rightarrow+\infty$. Therefore, both $E_{1}$ and $E_{2}$ are globally asymptotically stable. The proof of Theorem 3 is completed.

In the case when one of the microorganisms is going to vanish, some nonlinear oscillatory phenomena for the microorganism and the nutrient occur. In other words, in the corresponding stable manifold, a limit cycle exists. Regarding the dynamical system on the two dimensional faces $x=0$ or $y=0$, we have the following results.

For $E_{2}$, we study the phase portrait in the solution plane $x=0$. In this case the system (2) takes the form

$\frac{d S}{d t}=(1-S)-\frac{1}{C+D S_{0}^{3} S^{3}}\left(\frac{m_{2} S}{k_{2}+S}\right) y$

$\frac{d y}{d t}=\left(\frac{m_{2} S}{k_{2}+S}-1\right) y$

We would like to point out that the system (7) is a special case of the simple chemostat system:

$\frac{d S}{d t}=1-S-\frac{g(S)}{F(S)} y$

$\frac{d y}{d t}=(g(S)-1) y$

if letting $F(S)=C+D S_{0}^{3} S^{3}, g(S)=\frac{m_{2} S}{k_{2}+S}$.

In the region $\{(S, y) \mid 0 \leq S \leq 1, y \geq 0\}$, the system (8) has two steady state points $(1,0)$, and $\left(S^{*}, y^{*}\right)$ if $g(1)>1$, where

$y^{*}=\left(1-S^{*}\right) F\left(S^{*}\right), S^{*}=g^{-1}(1)$.

It is easy to see that $(1,0)$ is globally asymptotically stable if $g(1)<1$, unstable if $g(1)>1$. Denote 
$p=1+\left.y * \frac{d}{d S}\left(\frac{g}{F}\right)\right|_{S=S^{*}}$

The following theorem is established [4].

Theorem 4: Assume $g(1)>1$. If $p>0$ then $\left(S^{*}, \mathrm{y}^{*}\right)$ is stable; if $p<0$, it is unstable and there exists at least one limit cycle in (8) surrounding the equilibrium $\left(S^{*}, y^{*}\right)$.

Then, we have

Theorem 5: Assume (3) or $m_{2}>k_{2}+1$. The system (7) has two steady state points: $M_{1}(1,0)$, which is unstable, and $M_{2}\left(\lambda_{2},\left(1-\lambda_{2}\right)\left(C+D S_{0}^{3} \lambda_{2}^{3}\right)\right)$, which is stable if $C / D>R_{2}$, and unstable if $C / D<R_{2}$. In the case when $M_{2}$ is unstable, there exists at least one limit cycle in (7) surrounding $M_{2}$.

In the face $y=0$, the system (2) takes the form

$\frac{d S}{d t}=1-S-\frac{1}{A+B S_{0} S}\left(\frac{m_{1} S}{k_{1}+S}-L\right) x$

$\frac{d x}{d t}=\left(\frac{m_{1} S}{k_{1}+S}-L-1\right) x$

which is also a special case of (8) with

$F(S)=A+B S_{0} S, g(S)=\frac{m_{1} S}{k_{1}+S}-L$ and $y=x$

In $R_{+}^{2}=\{(S, x) \mid 0 \leq S \leq 1, x \geq 0\}$, the system (10) has steady state points: $N_{1}(1,0)$ which is unstable, and $N_{2}\left(\lambda_{1},\left(1-\lambda_{1}\right)\left(A+B S_{0} S\right)\right)$, if $g(1)>1$.

Calculating $p$ in (9) and $R_{1}$ will result the following theorem.

Theorem 6: Assume (3) or $\left.m_{1}>\left(k_{1}+1\right)(L+1)\right)$. If $A / B>R_{1}$, then $N_{2}$ is stable; If $A / B<R_{1}$, then $N_{2}$ is unstable and there exists at least one limit cycle in (10) surrounding $N_{2}$.

Regarding the bifurcation on the two dimensional stable manifolds, the following theorem is valid.

Theorem 7: The system (10) undergoes a Hopf bifurcation at $A / B=R_{1}$ and so does the system (7) at $C / D=R_{2}$.

Proof: Let $J\left(N_{2}\right)$ be the Jacobian at $N_{2}$. The corresponding characteristic equation is

$$
r^{2}+b_{1} r+c_{1}=0 .
$$

Let $A / B=\mu$. Denote $b_{1}$, the coefficient of $r$ in the above equation, as $\operatorname{tr}(A / B)$, or $\operatorname{tr} J(\mu)$, where,

$\operatorname{trJ}(\mu)=1+\left(1-\lambda_{1}\right)\left(\frac{-S_{0}}{\mu+\lambda_{1} S_{0}}+\frac{k_{1} m_{1}}{\left(k_{1}+\lambda_{1}\right)^{2}}\right)$.

Since

$\left.\frac{d}{d \mu} \operatorname{trJ}(\mu)\right|_{\mu=R_{1}}=\left(1-\lambda_{1}\right) \frac{S_{0}}{\left(R_{1}+\lambda_{1} S_{0}\right)^{2}}>0$,

the function $\operatorname{trJ}(\mu)$ is increasing at $\mu=R_{1}$.

Since

$$
\operatorname{trJ}(\mu) \begin{cases}<0 & \text { if } \mu<R_{1} \\ =0 & \text { if } \mu=R_{1} \\ <0 & \text { if } \mu>R_{1}\end{cases}
$$

the phase structure of $N_{2}=\left(\lambda_{1},\left(1-\lambda_{1}\right)\left(A+B \lambda_{1} S_{0}\right)\right)$ changes from unstable to stable at $R_{1}$ as the parameter $\mu$ increases. So (10) undergoes a Hopf bifurcation at $A / B=R_{1}$ by the definition.

Similarly, for the steady state point $M_{2}\left(\lambda_{2},\left(1-\lambda_{2}\right)(C+\right.$ $\left.D S_{0}^{3} \lambda_{2}^{3}\right)$ ), we can prove the bifurcation theorem for the system (7) at $C / D=R_{2}$.

\section{Numerical Simulations}

The numerical experiments performed on the system (2) using experimental data confirm our theoretical findings. For the parameter values $\quad m_{1}=3.25, k_{1}=2, m_{2}=1.75, k_{2}=$ $2.5, L=0.5, A=0.75, B=5, C=3.75, D=2.25, \quad S_{0}=$

0.4 , we have $\lambda_{1}>1, \lambda_{2}>1$ and the solution curves tend to $E_{0}$ (see Fig-1) representing the extinction of both microorganisms. For $m_{1}=3.25, k_{1}=0.2, m_{2}=2.5, k_{2}=$ $0.3, L=0.002 A=0.04, B=3, C=0.0001, D=0.2$,

$S_{0}=0.4$, we have $A / B>R_{1}, \lambda_{1}<\lambda_{2}$ and the solution curves tend to $E_{1}$ (see Fig-2) representing the extinction of second microorganism. For $m_{1}=3.5, k_{1}=0.4, m_{2}=3$, $k_{2}=0.2, L=0.001, A=0.0005, B=0.3, C=0.75$,

$D=3, S_{0}=0.4$, we have $C / D>R_{2}, \lambda_{1}>\lambda_{2}$ and the solution curves tend to $E_{2}$ (see Fig-3) representing the extinction of first microorganism. For $m_{1}=3.25, k_{1}=$ $0.2, L=0.002 A=0.0188496, B=2, S_{0}=0.4$, figures 45 contain the solution curves and phase structure of $N_{2}$ of the system (10), resulting the system undergoes a Hopf bifurcation at $A / B=R_{1}$. The two plots in figures-6,7 have $A / B>R_{1}$ and the the system (10) is unstable through limit cycle oscillation. The two plots in figure- 8,9 have $A / B<R_{1}$ and all plotted trajectories tend to $N_{2}$, resulting in a stable situation.

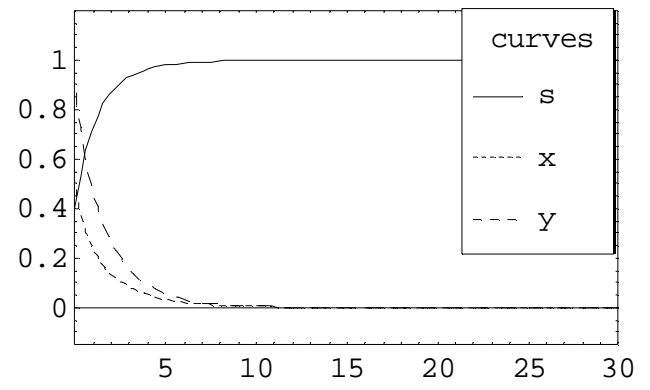

Fig. 1. Solution curves of (2) tend to $E_{0}$ 


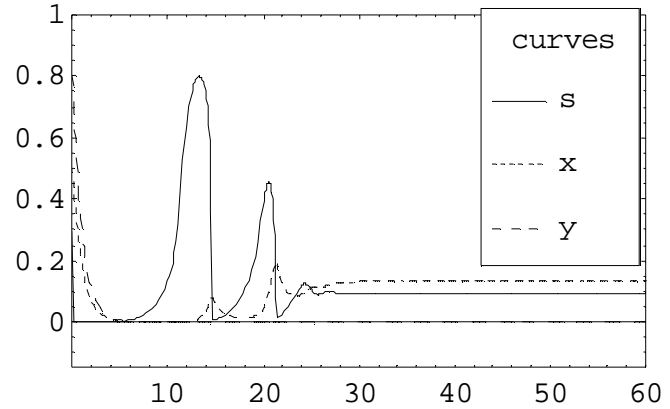

Fig. 2. Solution curves of (2) tend to $E_{1}$

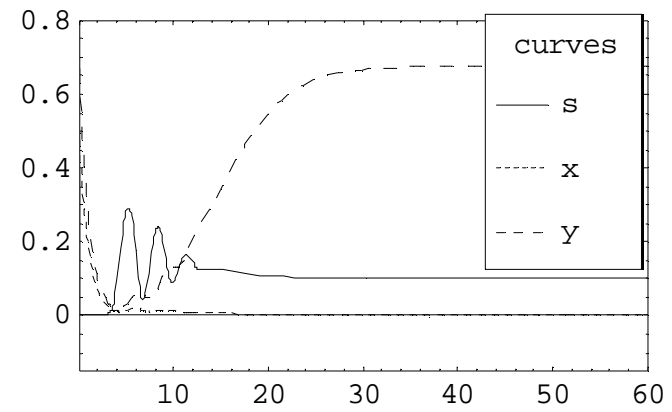

Fig. 3. Solution curves tend to $E_{2}$

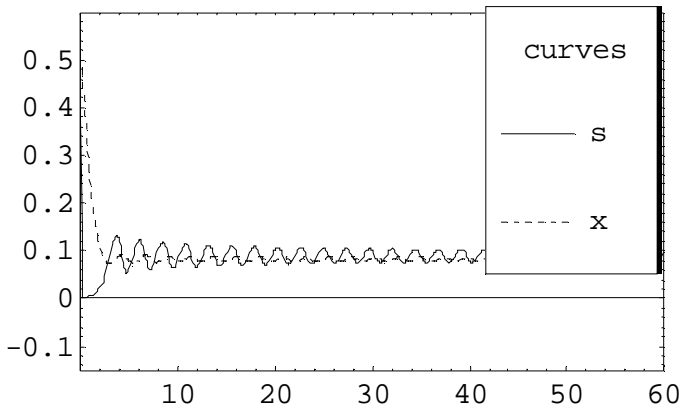

Fig. 4. Solution curves of system (10) when $A / B=R_{1}$

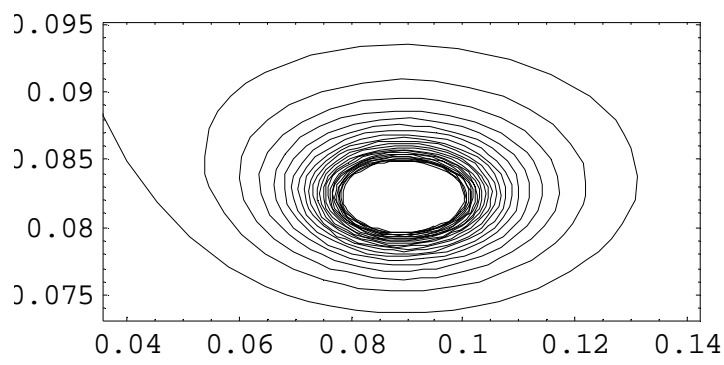

Fig. 5. System (10) undergoes a Hopf bifurcation at $A / B=R_{1}$

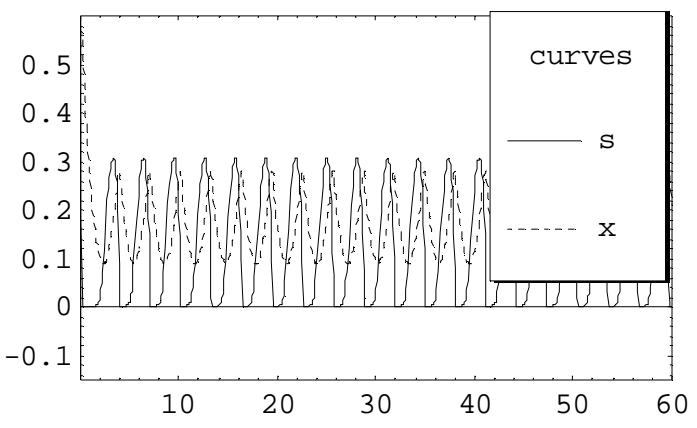

Fig. 6. Solution curves of system (10) when $A / B>R_{1}$

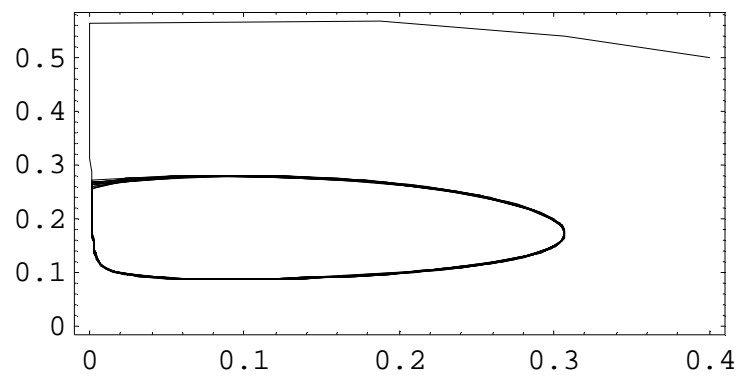

Fig. 7. System (10) is unstable when $A / B>R_{1}$

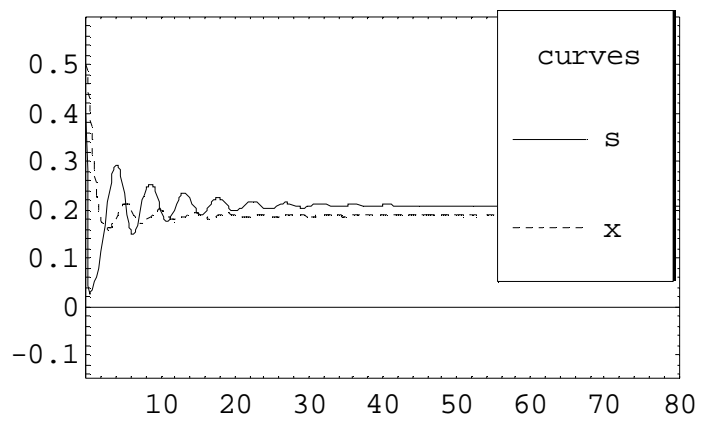

Fig. 8. Solution curves of system (10) when $A / B<R_{1}$

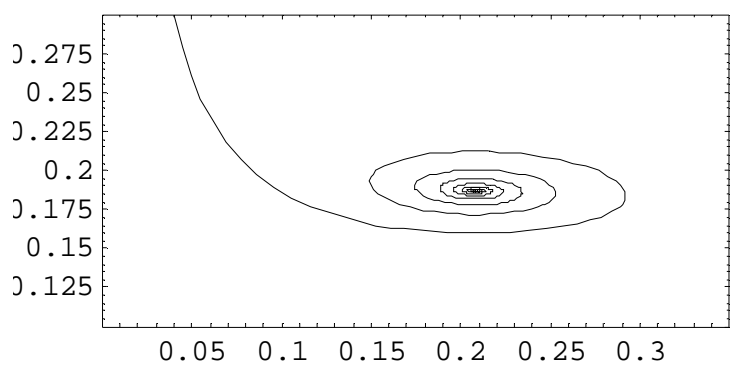

Fig. 9. System (10) is stable when $A / B<R_{1}$ 


\section{Conclusion}

In this paper, we considered a basic resource-based three dimensional chemostat model with variable yields. In this model, we assumed Monod type growth rate functions of which one yield coefficient is linear and another yield coefficient is cubic. The model (2) has three steady states. One is associated with extinction of both microorganisms, the other with survival of one microorganism. We studied the local stability of these steady states by using the Routh-Hurwitz criterion. We found that if the break-even concentration of one microorganism is larger than one then that microorganism dies out whether or not there is other competitor. If $E_{0}$ is the only steady state point, we showed that $E_{0}$ is LAS (this happens if both $\lambda_{1}>1$, $\left.\lambda_{2}>1\right)$. When $E_{0}, E_{1}$ or $E_{2}$ are the steady state points, we found that $E_{1}$ is LAS (for $A / B>R_{1}, \lambda_{1}<\lambda_{2}$ ) and $E_{2}$ is LAS (for $C / D>R_{2}, \lambda_{1}>\lambda_{2}$ ), i.e., the microorganism that survives is the one with the lowest break-even concentration.

Thus, for a variable yield coefficients chemostat model, we found that the break-even concentrations $\lambda_{1}, \lambda_{2}$ and parameters $A, B, C, D$ in variable yields play an important role for the extinction and survival of the microorganisms, and the limit cycle oscillation of the system.

1. Crooke P. S. and R. D. Tanner, 1982. Hopf bifurcations for a variable yield continuous fermentation model, Int.J. Eng. Sci. 20, 439-443.
2. Crooke P. S., C.J. Wei and R. D. Tanner, 1980. The effect of the specific growth rate and yield expressions on the existence of oscillatory behavior of the continuous fermentation model, Chem. Eng. Commun. 6, 333-339.

3. Dorofeev A.G., M.V. Glagolev, T.F. Bondarenko and N.S. Panikov, 1992. Observation and explanation of the unusual growth kinetics of Arthrobacter globiforms, Microbiology, 61, $33-42$

4. Huang X, 1990. Limit Cycles in a Continuous Fermentation Model, J.Math, Chemistry, 5, 287-296.

5. Huang X and L.M. Zhu, 2005. A three dimensional chemostat with quadratic yields, Journal of Mathematical Chemistry Vol. 38, No.4, November 575-588.

6. Huang $X$ and L.M. Zhu, 2005. Bifurcation in a Three Dimensional Continuous Fermentation Model, Int. J. Appl. Sci .and Eng.3, 2:117-123.

7. Pilyugin S. S. and P. Waltman, 2003. Multiple limit cycles in the chemostat with variable yield, Math. Biosci. 182, 151-166.

8. Rana S.M.S, A.F.M.K. Khan, 2010, Asymptotic Behavior of Single Species Chemostat Model, Dhaka Univ.J.Sci. 58(1): 127-131.

9. Song G. and X. Li, 1999. Stability of solution to the chemostat system with non-constant consuming rate, J. Biomath. 14:1, 24.

10. Smith H. L. and P. Waltman, 1995. The Theory of Chemostat. Cambridge University, Cambridge, UK.

11. Zhu, L. and X.C. Huang. 2005. Relative positions of limit cycles in a continuous culture vessel with variable yields. Journal of Mathematical Chemistry, 38, 2:119-128.

12. Zhu, L. and X.C. Huang. 2005. Multiple limit cycles in a continuous culture vessel Nonlinear analysis: Theory, Methods and Applications. (accepted). 